\title{
Eshelby-Kröner self-consistent elastic model: the geometric mean versus the arithmetic mean - A numerical investigation
}

\author{
S. Fréour and J. Fajoui \\ Institut de Recherche en Génie Civil et Mécanique (UMR CNRS 6183), LUNAM Université-Université de Nantes- \\ Centrale Nantes, CRTT, 37 Boulevard de l'Université, BP 406, 44602 Saint-Nazaire cedex, France
}

\begin{abstract}
Scale-transition models, such as Eshelby-Kröner self-consistent framework, which are often used for predicting the effec-tive behavior of heterogeneous materials or estimating the distribution of local states from the knowledge of the corre-sponding macroscopic quantities, require the extensive use of set averages. The present paper is devoted to the comparison of the numerical results provided in pure elasticity by Eshelby-Kröner model depending on the average type chosen for achieving set average operations: either the traditional arithmetic mean or the geometric average. Various numerical applications of the model to the case of predicting either the effective stiffness or the lattice strains of singlephase polycrystals will be provided. The particular case when an extreme grain-shape occurs will also be investigated.
\end{abstract}

Key words Scale transition model, sets averages, geometric mean, arithmetic mean, Eshelby-Kröner self-consistent model.

\section{Introduction}

An enhanced interpretation of the mechanical behaviour observed during experiments performed on polycrystalline samples can be achieved using scale transition models based on the description of interactions among the grains constituting the material and the polycrystal itself. Thus, these scale transition models were also recently called "grain-interaction models" [1].

Generally, sets averages are involved in any grain interaction model and required in order to determine the effective properties of the polycrystal from the properties of the differently oriented grains. The scale transition relations existing between the single grain/crystal properties and those of the polycrystals vary from one model to another. Historically, however arithmetic averages only were proposed and used to perform such calculations [2-8]. More recently, the idea of replacing arithmetic averages by geometric averages was proposed by Morawiec [9]. This new solution, introduced in particular cases by Aleksandrov and Aisenberg [10], is based on the condition of commutation of inversion and averaging operations that is one fundamental property observed in practice in materials sciences. One new scale transition model, the Bulk Path Geo (BPG) was recently built upon this constitutive assumption [11,12]. Numerical computations of the effective elastic behaviour of possibly textured polycrystals were achieved. It was shown by the authors that Young's modulus predicted by the BPG approximation was very close to the numerical values provided by Neerfeld-Hill or Eshelby-Kröner model using arithmetic averages.

Nevertheless, the Bulk Path Geo remains independent from any other model. Thus the closeness of its predictions with those of others models does not prove that geometric averages could be considered as a reliable alternative to the classical arithmetic averages in the field of computational materials science. This is of importance when experimental results have to be interpreted using micro-mechanical models in order to deduce mechanical constants like Single-crystal Elastic Constants (SEC). Until now, only the type of assumed grain-interactions was considered to play a role in such studies. But recently, SEC were determined using either geometric [13] or arithmetic averages, and the importance of the choice of such a set averaging method on the final result has to be investigated.

In the present work, Eshelby-Kröner self-consistent scale transition models involving either arithmetic averages or geometric averages will be used to predict the mechanical properties of various single-phase polycrystals in pure elasticity regime. The present paper derives from the recently published theoretical investigation of a product-based, "geometric", deviation of the mechanical states experienced by a single crystallite from the corresponding macroscopic quantities, achieved in [14]. Moreover, the influence of a morphologic texture will be considered through the introduction of extreme grain

* Corresponding author E-mail: sylvain.freour@univ-nantes.fr, Fax: +33240172618 
shapes in the computations. Besides, another section of the present paper is devoted to investigating the mechanical states experienced by the diffracting volume of polycrystals, depending on the chosen set average method: either the arithmetic or the geometric mean. The obtained results will be compared and discussed in order to conclude about the equivalence or not of the two, considered in this work, grain average types.

\section{Short summary of the theoretical framework}

\subsection{Definition of the different scales of the representation}

In the following, it is useful to distinguish three types of averages of a tensor $\mathbf{A}$ (as for example the strain tensor $\varepsilon$ ), that correspond to the three considered scales of the representation.

(i) Averages of a tensor over all grains with a particular crystallographic orientation in the volume considered: this average will be denoted by the symbol $\mathbf{A}^{\mathrm{II}}(\Omega)$, where $\Omega$ represents a vector in the three-dimensional crystallographic orientation (Euler) space and defines the crystallographic orientation (for details, see, for example, [15]. $\Omega=(\alpha, \beta, \gamma)$, where $\alpha, \beta$, and $\gamma$ are three Euler angles. The convention of Roe [16] for the definition of the Euler angles will be adopted (see also [15]). This average defines the behaviour of the material at the so-called "mesoscopic scale", denoted by the superscript II. Since the properties and mechanical states are identical for a given crystallographic orientation, there is no numerical operation to perform, in practice, in order to achieve the determination of any mesoscopic quantity.

(ii) Averages over diffracting grains: in polycrystalline materials, diffraction methods usually provide information probed from numerous grains. As a result, the gathered peak positions are average values [17-19]. However, since diffraction can occur only when the normal of these grains bisect the incident and diffracted beams in the classical case that monochromatic radiation is employed, the averaging does not occur over all of the grains in the irradiated volume, but over the particular subset of the crystallites for which the diffracting planes are perpendicular to the chosen measurement direction, instead. For a $h k l$ diffraction line, the group of diffracting crystallites is selected by specifying the $h k l$ of the reflection considered and the orientation of the diffraction vector with respect to the specimen reference frame, which can be identified by the angles $\varphi, \psi$ as defined on Fig. 1. For details, see also [20]. This type of average over the diffracting volume will be denoted by $\mathbf{A}^{D V}=\langle\mathbf{A}(\Omega)\rangle^{D V}$ where the superscript DV indicates the diffracting volume.

(iii) Averages over all crystallographically differently oriented grains in the polycrystal: this average will be denoted by angular brackets $\mathbf{A}^{\mathrm{I}}=\langle\mathbf{A}(\Omega)\rangle^{\mathrm{I}}$. This notation will be used to define the properties and mechanical states at the so-called I superscripted "macroscopic scale".

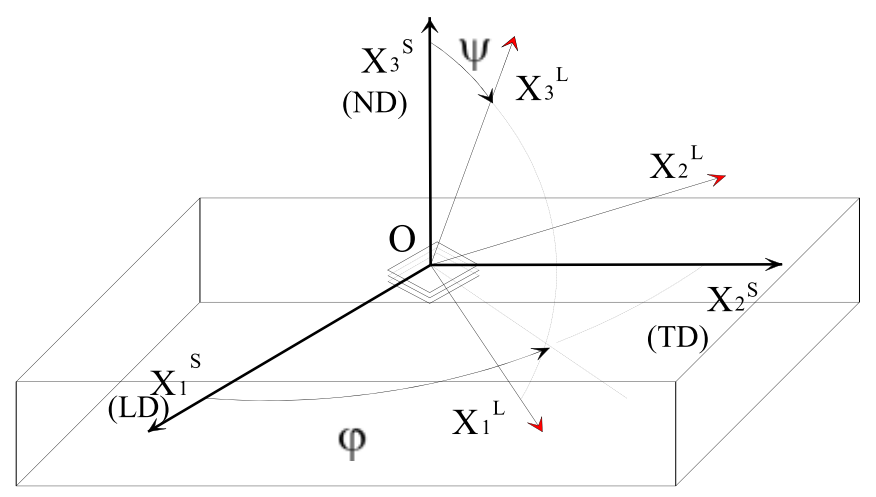

Fig. 1 (online colour at: www.zamm-journal.org) Definition of the angles $\phi$ and $\psi$ and orientation of the laboratory system $\mathrm{L}$ with respect to the sample system S. LD, TD, and ND, respectively, stands for Longitudinal, Transverse, and Normal Directions in the reference frame of the sample.

Two numerical methods dedicated to the determination of tensors averages over the polycrystal or the diffracting grains (respectively $\langle\mathbf{A}(\Omega)\rangle^{\mathrm{I}}$ and $\langle\mathbf{A}(\Omega)\rangle^{D V}$ ) will be described in Subsect. 2.2 below.

\subsection{Performing geometric and arithmetic averages}

Averages over sets of differently oriented grains can be performed using either the classical arithmetic approximation, or the recently proposed geometric approximation. Let us consider a subset of $N$ grains. Each $i$-subscripted grain ( $i$ varying from 1 to $N)$ has a specific orientation $\Omega_{i}=\left(\alpha_{i}, \beta_{i}, \gamma_{i}\right)$. That crystallographic subset actually corresponds to a particular volume fraction $f_{i}$ which is returned by the Orientation Distribution Function obtained through texture analysis.

The arithmetic approximation assumes that tensor averages correspond to the sum of the mesoscopic quantities, multiplied by the volume fraction $\mathrm{f}_{i}$ of each grain, over the considered volume (polycrystal in the case of the macroscopic scale), 
and divided by the total volume fraction of the considered subset of grains:

$$
\mathrm{AA}\left\langle\mathrm{A}^{\mathrm{I}}\right\rangle=\mathrm{AA}_{\mathrm{A}}\left\langle\mathrm{A}_{i}^{\mathrm{II}}\left(\Omega_{i}\right)\right\rangle_{i=1,2, \ldots, n, \ldots, N}=\alpha\left(\sum_{i=1}^{N} \mathrm{~A}_{i}^{\mathrm{II}}\left(\Omega_{i}\right) \mathrm{f}_{i}\right),
$$

where the subscript AA obviously stands for Arithmetic Average and $\alpha=\left(\sum_{i=1}^{N} f_{i}\right)^{-1}$.

In the case that the diffracting volume is concerned, only the grains contributing to the diffraction peak have to be taken into account in the summation.

The geometric mean of a set of weighted positive data is defined as the $f^{\text {th }}$ power of the product of all the members of the set:

$$
\mathrm{GA}\left\langle\mathrm{A}^{\mathrm{I}}\right\rangle=\mathrm{GA}\left\langle\mathrm{A}_{i}^{\mathrm{II}}\left(\Omega_{i}\right)\right\rangle_{i=1,2, \ldots, n, \ldots, N}=\left(\prod_{i=1}^{N}\left[\mathbf{A}_{i}^{\mathrm{II}}\left(\Omega_{i}\right)\right]^{f_{i}}\right)^{\alpha}
$$

In the case that the diffracting volume is concerned, only the grains to the diffraction peak have to be taken into account in the product.

The practical realization of such a geometric average on even rank tensor was detailed in $[9,21,22]$.

In Sect. 3, Eshelby-Kröner self-consistent elastic model featuring one or the other averaging type, will be studied. Numerical computations performed according to the geometric or the arithmetic approximations will be compared and discussed.

\subsection{Grain-shape/morphological texture}

For the calculation of mechanical and diffraction elastic constants of polycrystals with a grain-shape texture, the treatment will be restricted to polycrystals consisting of ellipsoidal grains. It will be assumed that the ellipsoidal grains exhibit identical orientations of their principal axes in the specimen frame of reference, i.e. the ellipsoidal grains are aligned along common axes (an ideal grain-shape texture occurs). Only an ideal grain-shape texture is considered in the following, as only in this case unique mechanical elastic constants and X-ray stress factors can be calculated employing the Eshelby-Kröner model (for a more detailed discussion of the effect of a non-ideal morphological texture, see [23]).

Note that the principal axes of a grain are only related to the external (geometrical) shape of the grain. Thereby nothing is prescribed regarding the crystallographic orientation of the grain (crystallite). In general, the (ellipsoidal) grains constituting the specimen will have different crystallographic orientations.

The shape of the grains will be described by a shape parameter $\eta$, which is defined as the ratio of the principal axis of the ellipsoid in the $z$-direction $\left(a_{3}\right)$ of the specimen frame of reference to the principal axes of the ellipsoid in the $x$-direction $\left(a_{1}\right)$ and the $y$-direction $\left(a_{2}\right)$ in the specimen frame of reference, respectively:

$$
\eta=\frac{a_{3}}{a_{1}}=\frac{a_{2}}{a_{1}} .
$$

Thus, the considered ellipsoidal grains present rotational symmetry with respect to the surface normal of the specimen.

\section{Modelling the elastic behaviour of polycrystals according to Eshelby-Kröner self-consistent model - comparison between geometric and arithmetic averaging}

\subsection{Eshelby-Kröner self-consistent elastic model}

\subsubsection{Determination of the macroscopic elastic stiffness according to Eshelby-Kröner hypotheses}

In order to calculate the elastic constants of a polycrystal from single-crystal elastic data, the crystallites surrounding an individual grain in a polycrystal are conceived as an elastically homogenous matrix with the elastic properties of the entire polycrystal in the Eshelby-Kröner grain-interaction model [6,7]. Following this reasoning, the calculation of the elastic constants of a polycrystal requires the calculation of the elastic interactions existing between an inclusion (a grain) and the embedding homogeneous matrix (the polycrystal). Traditionally, a spherical shape of the inclusion is considered [7]. It goes without saying that the Eshelby-Kröner model based on a spherical inclusion will only work for polycrystals consisting of (on average) spherical, equi-axed grains.

The effect of a grain-shape (morphological) texture on mechanical and diffraction elastic constants can be considered in the traditional Eshelby-Kröner model by considering ellipsoidal inclusions with their principal axes aligned along common 
directions in the specimen frame of reference. While Eshelby-Kröner model can handle a single grain-morphology, shared by every inclusion constituting the Representative Equivalent Volume (REV) of the material, this theoretical approach fails to represent a morphologic texture featuring various grain shapes and/or a relative disorientation of the morphologies coexisting among the same REV. It was actually numerically shown by [24], that in such a situation, the classical EKSC model does not simultaneously fulfil both the so-called "Hill's average relations over the mechanical states", historically established in [25], anymore. An enhanced version of the classical Eshelby-Kröner self-sonsistent model, the so-called "Generalized Self-Consistent" (GSC) model, is required in order to properly deal with a real morphologic microstructure. As an example, the structure of Ti-17 polycrystal often consists of acicular $\alpha$ (needle-shaped crystallites) mixed to slightly equiaxed prior $\beta$ grains. The morphologic microstructure exhibited by Ti-17 $\alpha$-phase cannot be properly taken into account through the traditional EKSC model. A recent investigation taking advantage of the GSC model, however, was specifically built in order to model such a material [26].

Nevertheless, the present study is based on a demonstration, provided by [14], established within the classical EshelbyKröner model domain of application. As a consequence, this paper will only deal with polycrystals made of ellipsoidal inclusions with their principal axes aligned along common directions in the specimen frame of reference. To characterise the grain-shape texture, the convention introduced in Subsect. 2.3 (Grain-shape/morphological texture) will be adopted. The effect of a grain-shape texture on the mechanical elastic constants has been considered by [23, 27, 28].

Within Eshelby-Kröner model, the macroscopic stiffness $\mathbf{C}^{\mathrm{I}}$ is calculated iteratively through an averaging procedure over every crystallite as follows:

$$
\mathbf{C}^{\mathrm{I}}=\left\langle\mathbf{C}^{\mathrm{II}}(\Omega):\left[\mathbf{E}^{\mathrm{I}}:\left(\mathbf{C}^{\mathrm{II}}(\Omega)-\mathbf{C}^{\mathrm{I}}\right)+I\right]^{-1}\right\rangle .
$$

Where $\mathbf{C}^{\mathrm{II}}(\Omega)$ stands for the single-crystal elastic stiffness, $\mathbf{I}$ is the 4th-order Identity tensor, whereas $\mathbf{E}^{\mathrm{I}}$ is Morris tensor, which can be written from the Eshelby tensor $\mathbf{S}_{\text {esh }}^{\mathrm{I}}$ thanks to the following relation:

$$
\mathbf{E}^{\mathrm{I}}=\mathbf{S}_{\mathrm{esh}}^{\mathrm{I}} \mathbf{C}^{\mathrm{I}^{-1}}
$$

Morris tensor is written as an integral that must be numerically computed, except for a few simplified cases. The interested reader can refer to [28-30], where expressions of Morris tensor as well as a detailed presentation of Eshelby-Kröner model, are provided. Because of the complex expression of the integrand featured within Morris tensor, one cannot generally give an analytical expression of $\mathbf{E}^{\mathrm{I}}$, except for some specific configurations (fibers, discs and spheres in particular). The case of macroscopically transversely isotropic materials made of either aligned fibers parallel to the longitudinal direction or flat-discs the normal of which is parallel to the normal direction of the sample, were, as an example extensively treated in $[31,32]$.

The above presented fundamental equation (4) is actually compatible with either the historical, classical arithmetic framework or, as demonstrated for the first time in [14], with a product-based, "geometric" rewriting of the polarization tensors used to express the deviation of the mechanical states experienced by a single crystallite from the corresponding macroscopic quantities. The first formulation results in a sum of terms, whereas the second involves products of factors. This fact yields a privileged (but not exclusive) link between each formulation and the corresponding averaging operationtype. It was demonstrated in [14] that the geometric polarization tensors are proportional to either the strain localization tensor or the stress concentration tensor, two quantities on which many scale transition models are based upon, for practical applications, but also because of their strong physical meaning. On the contrary, the classical arithmetic polarization tensors did hold very little appeal on the scientific community working on this field of research. Since the arithmetic mean is considered as better suited for achieving averages over sets of sums of terms, it was historically clearly the most appropriate solution, in the mathematical framework of Eshelby-Kröner model. However, according to [14] the geometric set averagetype, which is, in statistics, considered as better suited for performing such mathematical operations over products of factors, would also be relevant, since the corresponding analytical writing of Eshelby-Kröner model involves more physically meaningful intermediate quantities. Thus, according to the results obtained in [14], either the arithmetic, or the geometric mean, could be employed at the discretion of the user in order to perform calculations according to Eshelby-Kröner selfconsistent model.

\subsubsection{Computation of the lattice strains according to Eshelby-Kröner model}

$\mathrm{X}$-Ray stress analysis is an experimental method that enables the determination of the residual [19] or applied [33] average stress in the diffracting volume of a studied phase of a material from the measurement of the so-called (elastic) "lattice strains". The lattice strains $\varepsilon_{\varphi \psi}(h k l)$ actually correspond to the average of the mesoscopic strains over the grains suitably 
aligned to the X-ray beam path (as described in Subsects. 2.1 and 2.2). The projection of the mesoscopic strains on the measurement direction is defined by the two angles $\varphi$ and $\psi$ as shown on Fig. 1 [19]:

$$
\varepsilon_{\phi, \psi}^{D V}=\vec{n}^{t} \cdot\left\langle\varepsilon^{\mathrm{II}}(\Omega)\right\rangle^{D V} \cdot \vec{n}, \quad \text { with } \quad n=\left(\begin{array}{c}
\sin \psi \cos \varphi \\
\sin \psi \sin \varphi \\
\cos \psi
\end{array}\right) .
$$

In relation (6) above, the superscript $t$ denotes the transposition of the vector.

The computation of lattice strains, following the classical arithmetic averaging procedure is straightforward, as indicated in Subsect. 2.2. In the case that the geometric average is preferred, some precautions have to be considered since the mesoscopic strains experienced by the grains contributing to the diffraction may generally have some negative components, which are incompatible with the application of the power product (2) necessary to achieve the calculation of (6):

- First, the classical scale transition relation linking the mesoscopic strains to the macroscopic strains within EshelbyKröner self-consistent model (refer to [34] for details) has to be considered:

$$
\varepsilon^{\mathrm{II}}(\Omega)=\left[\mathbf{E}^{\mathrm{I}}:\left(\mathbf{C}^{\mathrm{II}}(\Omega)-\mathbf{C}^{\mathrm{I}}\right)+I\right]^{-1}: \varepsilon^{\mathrm{I}} .
$$

- Then, (7) is introduced in Eq. (6), which transforms as follows:

$$
\varepsilon_{\phi, \psi}^{D V}=\vec{n}^{t} \cdot\left\langle\left[\mathbf{E}^{\mathrm{I}}:\left(\mathbf{C}^{\mathrm{II}}(\Omega)-\mathbf{C}^{\mathrm{I}}\right)+I\right]^{-1}: \varepsilon^{\mathrm{I}}\right\rangle^{D V} \cdot \vec{n}
$$

The term $\varepsilon^{\mathrm{I}}$ being constant, it can be factorized in the average over the diffracting volume featured in the right-hand member of Eq. (8):

$$
\varepsilon_{\phi, \psi}^{D V}=\vec{n}^{t} \cdot\left\langle\left[\mathbf{E}^{\mathrm{I}}:\left(\mathbf{C}^{\mathrm{II}}(\Omega)-\mathbf{C}^{\mathrm{I}}\right)+I\right]^{-1}\right\rangle^{D V}: \varepsilon^{\mathrm{I}} \cdot \vec{n}
$$

In (8), the lattice strain over the diffracting volume is actually written under the form of the average of the elementary mesoscopic lattice strains $\varepsilon_{\phi, \psi}^{\mathrm{II}}(\Omega)$ of each crystallite contributing to the diffracted beam. Such a mesoscopic lattice strain would satisfy:

$$
\varepsilon_{\phi, \psi}^{\mathrm{II}}=\vec{n}^{t} \cdot\left[\mathbf{E}^{\mathrm{I}}:\left(\mathbf{C}^{\mathrm{II}}(\Omega)-\mathbf{C}^{\mathrm{I}}\right)+I\right]^{-1}: \varepsilon^{\mathrm{I}} \cdot \vec{n}
$$

$\varepsilon_{\phi, \psi}^{\mathrm{II}}(\Omega)$ is obviously a scalar. In practice, when the applied load is sufficiently high, the sign of all the mesoscopic lattice strains is often identical on the subset of grains constituting the diffracting volume, whatever the concerned crystallographic orientations. While this condition is satisfied, the power product involved by the geometric mean can be applied to the following rewritten form of Eq. (9):

$$
\varepsilon_{\phi, \psi}^{D V}=\operatorname{sign}\left[\varepsilon_{\phi, \psi}^{\mathrm{II}}(\Omega)\right]\left|\vec{n}^{t} \cdot\left\langle\left[\mathbf{E}^{\mathrm{I}}:\left(\mathbf{C}^{\mathrm{II}}(\Omega)-\mathbf{C}^{\mathrm{I}}\right)+I\right]^{-1}\right\rangle^{D V}: \varepsilon^{\mathrm{I}} \cdot \vec{n}\right|,
$$

where $\operatorname{sign}\left[\varepsilon_{\phi, \psi}^{\mathrm{II}}(\Omega)\right]$ is respectively -1 in the case that each mesoscopic lattice strain (10) involved in the averaging operation (11) is compressive, and +1 , in the case that each of the diffracting grains experience tensile lattice strains in the probed $\{\varphi \psi\}$ direction. Please take care of the fact that the geometric average cannot be computed in the case that both tensile and compressive lattice strains are simultaneously experienced by the subset of grains constituting the diffracting volume of the material.

\subsection{Examples - numerical comparisons between geometric and arithmetic averaging}

\subsubsection{Prediction of macroscopic elasticity constants in single-phase materials. Investigation of grain-shape effects}

Computations of the macroscopic elastic stiffness were performed following the formalism described in Subsect. 3.1, using either the arithmetic or the geometric averages. In order to avoid statistical errors, the same set of 2000 crystallographic orientations was kept for every calculation. Moreover the materials were considered as crystallographically untextured. 
Thus the volume fraction of each orientation was assumed to be identical. In order to check the possible morphological texture related effects, three extreme grain shapes were successively considered during the computations: spherical grains $(\eta=1)$, disc-shaped grains $(\eta \rightarrow 0)$ and, finally, needle-shaped grains $(\eta \rightarrow \infty)$.

In order to iteratively calculate the stiffness tensor using (4), a starting value is required for $\mathbf{C}^{\mathrm{I}}$. According to the literature, elastic stiffness predicted by Eshelby-Kröner model involving arithmetic averaging respectively converges towards Neerfeld [4] - Hill [5], Vook-Witt [35] and inverse Vook-Witt [36] estimations, in the cases that equi-axed (spherical), discshaped, and needle (cigar)-shaped grains, respectively, are considered [32,37]. Thus, the three last-cited approximations were used in the present work in order to provide adequate starting value of $\mathbf{C}^{\mathrm{I}}$ for the considered morphologies.

The single-crystal elastic constants required for performing the computations were taken from the tables provided by Chung and Buessem [38].
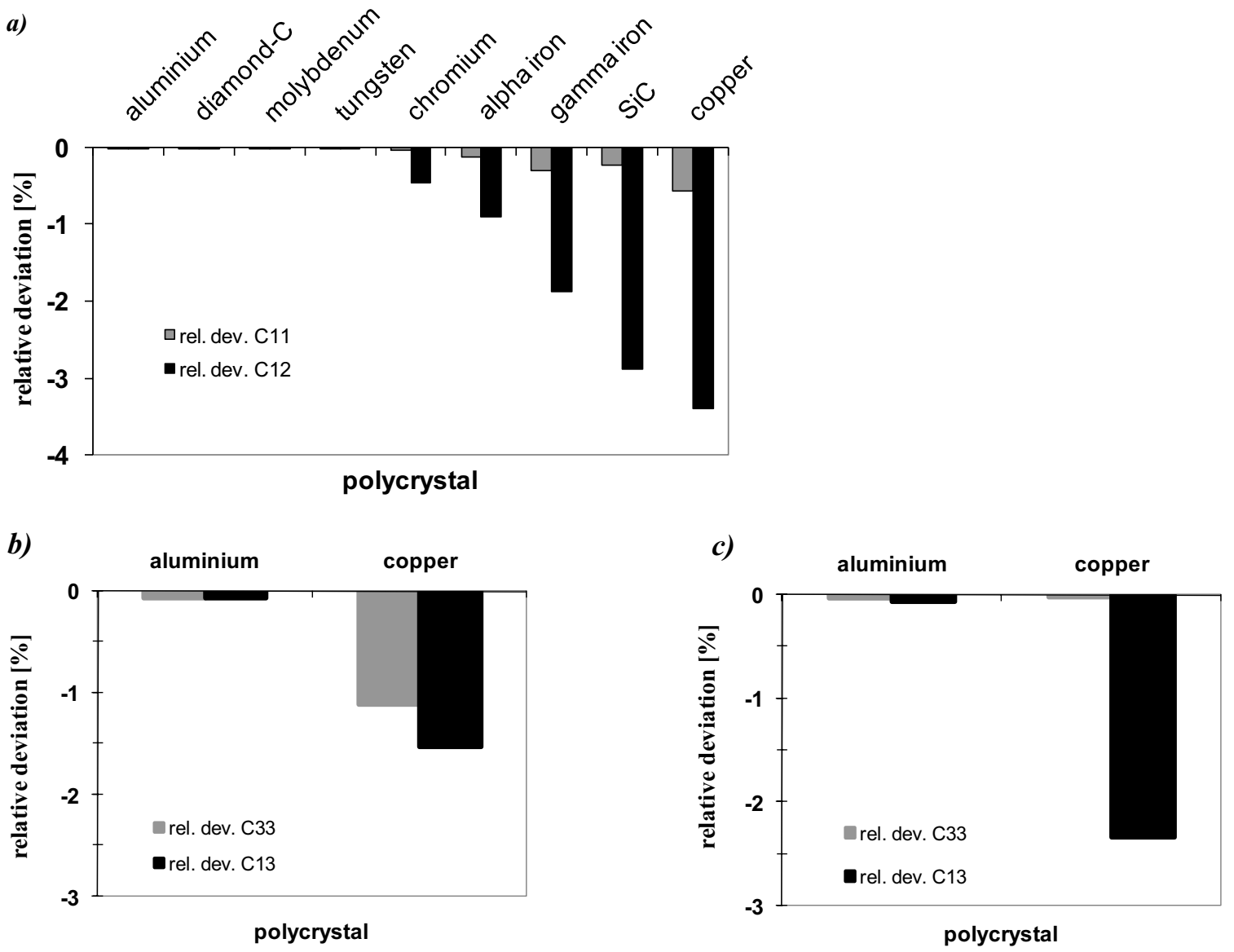

Fig. 2 Comparison between some macroscopic elastic stiffness components estimated with Eshelby-Kröner model involving either geometric or arithmetic averages for various materials. Study of grain shape effects: a) spherical grains, b) disc-shaped grains, and c) cigar-shaped grains.

Obtained results are depicted on Fig. 2, where the relative deviation between stiffness components calculated according to the geometric and the arithmetic approach (i.e. $\frac{\left.C_{i j}^{\mathrm{I}} \text { (geometric }\right)-C_{i j}^{\mathrm{I}} \text { (arithmetic) }}{\left.C_{i j}^{\mathrm{I}} \text { (arithmetic }\right)}$ ) are given for various single-phase cubic polycrystals.

Table 1 Coefficient of elastic anisotropy of various single-crystals [38].

\begin{tabular}{|l|l|l|l|l|l|l|l|l|l|}
\hline Crystal & $\mathrm{Al}$ & Diamond-C & $\mathrm{Mo}$ & $\mathrm{W}$ & $\mathrm{Cr}$ & $\alpha \mathrm{Fe}$ & $\gamma \mathrm{Fe}$ & $\mathrm{SiC}$ & $\mathrm{Cu}$ \\
\hline $\mathrm{A}_{c}$ & 1.23 & 1.00 & 0.91 & 0.97 & 0.71 & 2.41 & 3.34 & 2.20 & 3.21 \\
\hline
\end{tabular}


The following properties are remarkable:

(i) Geometric and arithmetic averages do not generally lead to identical macroscopic stiffness tensors.

(ii) Components of the stiffness tensor estimated through geometric mean are more compliant than (i.e. their numerical value is always inferior to) the corresponding component deduced from the classical arithmetic average.

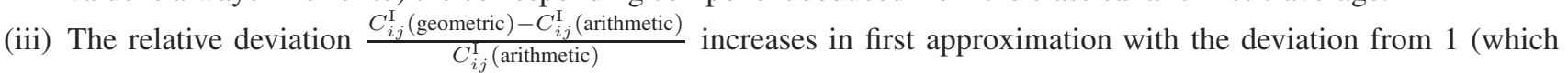
correspond to the elastic isotropy) of the considered material's single-crystal coefficient of elastic anisotropy $\mathrm{A}_{c}$ (cf. Table 1). Conversely, the relative deviation between stiffness components calculated according to the two studied averaging type tends towards zero in the cases that the single-crystal exhibits an almost isotropic elastic behaviour (aluminum, tungsten, molybdenum and diamond-C). This result was attributed to the relatively weak heterogeneities existing between the mechanical elasticity constants exhibited by the grains constituting a single-phase polycrystal. For metallic single-phase materials, the elastic heterogeneities existing at the mesoscopic scale are actually only induced by the coefficient of elastic anisotropy of the single crystal, and remain rather small. This property of single-phase metallic polycrystals explains the good agreement observed for the estimated effective macroscopic stiffness whatever the choice of averaging type, in the case that the material is assumed to deform according to purely elastic mechanism (Fig. 2).

(iv) The relative deviation between stiffnesses calculated according to the arithmetic or geometric mean strongly depends on the grain shape assumed during the computations. For instance, $C_{33}^{\mathrm{I}}$ values estimated for disc-shaped grains are almost identical with the two types of averages, even for materials having strongly anisotropic single-crystals like copper; whereas the same component calculated assuming cigar-shaped grains noticeably varies from one average type to another. Grain-shape texture induces a change in the distribution of the mechanical elastic heterogeneities experienced by a single-phase polycrystal at mesoscopic scale. The interested reader can refer to [32] where that specific issue was extensively investigated. Nevertheless, the elastic stiffness heterogeneities remains of the same order of magnitude whatever the considered extreme grain-morphology, according to Fig. 2. Thus, the predicted macroscopic stiffness of a single-phase polycrystal is almost independent on the set-average method chosen in order to achieve the computations, even in the case that an extreme grain-morphology is accounted for.

\subsubsection{Influence of the chosen averaging type on the elastic mechanical behavior calculated for the diffracting volume of the material}

Lattice strains in single-phase SiC corresponding to an applied macroscopic load of $100 \mathrm{MPa}$ in the longitudinal direction of the sample reference frame were calculated using Eshelby-Kröner model for various conditions. Three different calculation procedures were envisaged:

1) Full arithmetic approach. Both macroscopic stiffnesses (4) and average lattice strains (9) are calculated using the arithmetic mean (1).

2) Full geometric approach. Same as case 1), but applying the geometric mean (2) in equations (4) and (11), whether the macroscopic stiffness or the lattice strains have to be computed, respectively.

3) A mixed geometric-arithmetic procedure. The geometric mean (2) is kept for the calculation of the macroscopic stiffness (4) of the polycrystal that is required for computing Eq. (9). But, the lattice strains are calculated using the arithmetic average (1), in order to avoid indeterminations occurring with the geometric mean when mesoscopic elastic anisotropy leads to simultaneous positive and negative values of the mesoscopic lattice strains in the measurement direction (in that case, as explained in Subsect. 3.1.2, the arithmetic mean only enables calculation of the lattice strains over the diffracting volume of the material).

The assumed measurement conditions were as follows: $\varphi=0^{\circ} \psi$ varies from $0^{\circ}$ up to $90^{\circ}$ and the calculations were repeated for two diffracting planes, $\{200\}$ that does not present any mesoscopic elastic anisotropy (thus, the mechanical states and lattice strains are identical in one given measurement direction $\{\varphi \psi\}$ ) and the $\{311\}$ family, that generally presents a distribution of the mesoscopic mechanical states over the diffracting grains.

The obtained results are shown on Fig. 3. The following properties have been observed:

(v) No significant discrepancy of the lattice strains plotted as a function of $\sin ^{2} \psi$ appears in the case that single-phase materials are considered, whatever the choice of averaging type.

(vi) According to Fig. 3, a rather good agreement between the "mixed geometric-arithmetic" and the "full geometric" calculation schemes is obtained. This property is interesting because it provides a practical way to achieve realistic calculations of averaged mechanical states, taking into account the effect of the geometric mean on the effective 
a)

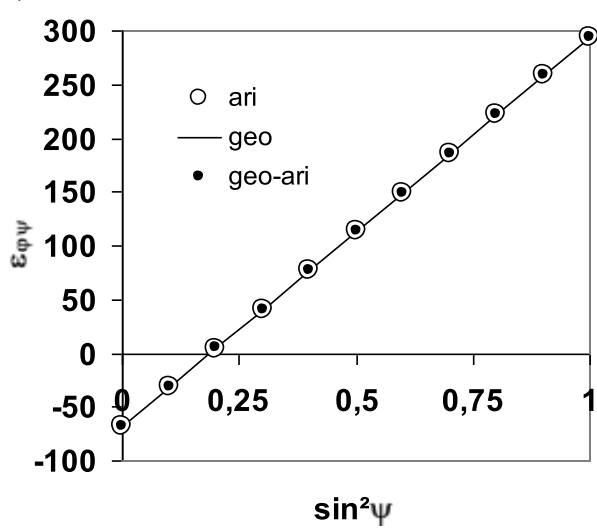

b)

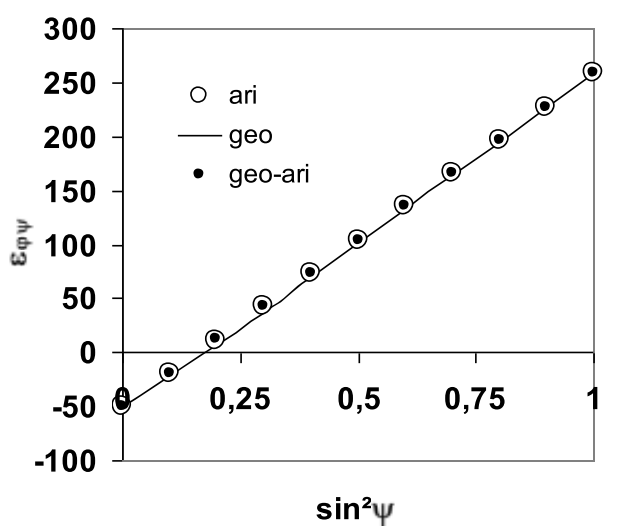

Fig. 3 Comparisons between lattice strains $\left[10^{-6}\right]$ estimated in silicon carbide with Eshelby-Kröner model involving either geometric or arithmetic averages. The captions ari, geo and geo-ari respectively stand for the "full arithmetic", "full geometric", and "mixed geometric-arithmetic" as described in Sect. 3.2.2.

mechanical behaviour of the polycrystal, without being limited by the mathematical constraints due to the powerproduct for the purpose of averaging mechanical states such as lattice strains.

\section{Conclusions and perspectives}

The present paper was devoted to the comparison of the numerical results provided in pure elasticity by Eshelby-Kröner model depending on the average type chosen for achieving set average operations: either the traditional arithmetic mean or the geometric average, on the basis of the theoretical analysis previously presented in [14]. Various numerical applications of the model to the case of predicting either the effective stiffness or the lattice strains of single-phase polycrystals were provided. The particular case when an extreme grain-shape occurs was also taken into account. It was shown that a mixed geometric-arithmetic set-average based methodology could be used in order to overcome the difficulties encountered when performing averaging operations over subsets of mechanical states including both positive and negative values.

According to the observations i) to vi) listed in Subsect. 3.2, the present work confirms that the micro-mechanical elastic models based on arithmetic or geometric averages are, in most cases, at least numerically close together, provided that single-phase materials are considered.

Thus, in order to treat such a case, the choice of an averaging type will have, at worst, only a minor, and, thus, by comparison to the experimental errors, negligible influence on the calculated behaviour of the studied polycrystal. It means, for instance, that the inversion of scale transition models, in order to estimate the single-crystal elastic properties from measurements performed on single-phase polycrystals, should lead to the determination of the same physical constants independently of the method chosen in order to perform averaging operations (knowing that the standard relative deviation on each estimated single-crystal elasticity constant is around 10\%, due to experimental errors). This is an important property, since both averaging types have been used in the literature: the classical application of the arithmetic mean to estimate single-crystal elasticity constants [39-41] having been recently, sometimes, replaced by the geometric mean [13].

Further studies will be devoted to an extensive comparison between the materials properties predicted by Eshelby-Kröner elastic model depending on the average type chosen for achieving set average operations, in the context of identifying the XRay Elastic Constants (XEC) or the Single-crystal Elastic Constants (SEC), from lattice strains measured on polycrystalline samples owing to diffraction methods. The aim would be to extend the results already obtained by [21], in the specific context of computing the Diffraction Elastic Constant (DEC) $\mathrm{F}_{11}$ of a strongly textured cold rolled ultra low carbon steel sample through various simple yet effective models (Reuss, Voigt, Hill) owing to through either the arithmetic average or the so-called "new average" proposed by Morawiec [9], which indeed corresponds to the geometric mean. The authors obtained a good agreement between the DEC predicted by a given model, whatever the considered set averaging method. However, the consequences related to that choice on the identified SEC has still not been investigated.

Extensions to the present analysis to the scale transition analysis of heterogeneous materials experiencing thermal or hygroscopic loads or even chemical shrinkage through the self-consistent model will also be provided in a forthcoming paper. 


\section{References}

[1] U. Welzel and E. J. Mittermeijer, Diffraction stress analysis of macroscopically elastically anisotropic specimens: On the concepts of diffraction elastic constants and stress factors, J. Appl. Phys. 93, 9001-9011 (2003).

[2] A. Reuss, Berechnung der Fliessgrenze von Mischkristallen auf Grund der Plastizitätsbedingung für Einkristalle, Z. Angew. Math. Mech. 9, 49-58 (1929).

[3] W. Voigt, Lehrbuch der Kristallphysik (Teubner, Leipzig/Berlin, 1928).

[4] H. Neerfeld, Zur Spannungsberechnung aus Röntgenographischen Dehnungsmessungen, Mitt. Kaiser-Wilhelm-Institut für Eisenforschung Düsseldorf 24, 61-70 (1942).

[5] R. Hill, The elastic behaviour of a crystalline aggregate. Proc. Phys. Soc. 65, 349-354 (1952).

[6] J. D. Eshelby, The determination of the elastic field of an ellipsoidal inclusion and related problems, Proc. R. Soc. Lond. A 241, 376-396 (1957).

[7] E. Kröner, Berechnung der elastischen Konstanten des Vielkristalls aus des Konstanten des Einkristalls, Z. Phys. 151, 504-518 (1958).

[8] G. Kneer, Über die Berechnung der Elastizitätsmoduln vielkristalliner Aggregate mit Textur, Phys. Stat. Solidi 9, 825—838 (1965).

[9] A. Morawiec, Calculation of polycrystal elastic constants from single-crystal data, Phys. Stat. Solidi B 154, 535-541 (1989).

[10] K. S. Aleksandrov and L. A. Aisenberg, A method for the computation of the physical constants of polycrystalline materials (in Russian), Dokl. Akad. Nauk SSSR 167, 1028-1031 (1966).

[11] S. Matthies and M. Humbert, The realization of the concept of a geometric mean for calculating physical constants of polycrystalline materials, Phys. Stat. Solidi B 177, K47-K50 (1993).

[12] S. Matthies, M. Humbert, and Ch. Schuman On the use of the geometric mean approximation in residual stress analysis, Phys. Stat. Solidi B 186, K41-K44 (1994).

[13] S. Matthies, S. Merkel, H. R. Wenk, R. J. Hemley, and H. Mao, Effects of texture on the determination of elasticity of polycrystalline $\varepsilon$-iron from diffraction measurements, Earth Planet. Sci. Lett. 194, 201-212 (2001).

[14] S. Fréour, E. Lacoste, J. Fajoui, and F. Jacquemin, On the meaning of the chosen set-averaging method within Eshelby-Kröner self-consistent scale transition model: the geometric mean versus the classical arithmetic average, Z. Angew. Math. Mech. 91(8), 689-698 (2011)

[15] M. Leoni, U. Welzel, P. Lamparter, E. J. Mittermeijer, and J.-D. Kamminga, Diffraction analysis of internal strain-stress fields in textured transversely-isotropic thin films: theoretical basis and simulation, Philos. Mag. A 81(3), 597-623 (2001).

[16] R. J. Roe, Description of crystallite orientation in polycrystalline materials III. General solution to pole figure inversion, J. Appl. Phys. 36(6), 2024-2031 (1965).

[17] I. C. Noyan and J. B. Cohen, Residual Stress. Measurement by Diffraction and Interpretation (Springer-Verlag, New York, 1987).

[18] M. Ortiz and A. A. Pochettino, Intergranular thermal stresses in zirconium - effects on X-rays macrostress measurements, J. Nucl. Mater. 229, 65-72 (1996).

[19] V. Hauk, Structural and Residual Stress Analysis by Nondestructive Methods, edited by V. Hauk (Elsevier, Amsterdam, 1997).

[20] S. Fréour, D. Gloaguen, M. François, and R. Guillén, Thermal properties of polycrystals - X-ray diffraction and scale transition modelling, Phys. Stat. Solidi A 201, 59-71 (2004).

[21] A. Baczmanski, K. Wierzbanowski, W. J. Haije, R. B. Helmoldt, G. Ekambaranathan, and B. Pathiraj, Diffraction elastic constants for textured materials - different methods of calculation, Cryst. Res. Technol. 28, 229-243 (1993).

[22] S. Matthies and M. Humbert, On the principle of a geometric mean of even-rank symmetric tensors for textured polycrystals, J. Appl. Crystallogr. 28, 254-266 (1995).

[23] N. Koch, U. Welzel, H. Wern, and E. J. Mittermeijer, Mechanical elastic constants and diffraction stress factors of macroscopically elastically anisotropic polycrystals: the effect of grain-shape (morphological) texture, Philos. Mag. 84(33), 3547-3570 (2004).

[24] E. Lacoste, S. Fréour, and F. Jacquemin, On the validity of the Kröner-Eshelby scale transition model for inclusion with various morphologies, Mech. Mater. 42, 218-226 (2010).

[25] R. Hill, The essential structure of constitutive laws for metal composites and polycrystals, J. Mech. Phys. Solids 15, 79-95 (1967).

[26] S. Fréour, E. Lacoste, M. François, and R. Guillén, Determining Ti-17 $\beta$-phase single-crystal elasticity constants through X-ray diffraction and inverse scale transition model, Mater. Sci. Forum 681, 97-102 (2011b).

[27] B. C. Hendrix and L. G. Yu, Self-consistent elastic properties for trans-versely isotropic polycrystals, Acta Materialia 46, 127-135 (1998).

[28] U. F. Kocks, C. N. Tomé, and H. R. Wenk, Texture and Anisotropy, (Cambridge University Press, Cambridge, 1998).

[29] R. J. Asaro and D. M. Barnett, The non-uniform transformation strain problem for an anisotropic ellipsoidal inclusion, J. Mech. Phys. Solids 23, 77-83 (1975).

[30] T. Mura, Micromechanics of Defects in Solids (Martinus Nijhoff Publishers, The Hague, 1982).

[31] S. Fréour, F. Jacquemin, and R. Guillén, On an analytical self-consistent model for internal stress prediction in fiber-reinforced composites submitted to hygro-elastic load, J. Reinf. Plast. Compos. 24, 1365-1377 (2005).

[32] U. Welzel, S. Fréour, and E. J. Mittermeijer, Direction-dependent elastic grain-interaction models - a comparative study, Philos. Mag. 85, 2391-2414 (2005).

[33] S. Fréour, D. Gloaguen, M. François, A. Perronnet, and R. Guillén, Determination of single-crystal elasticity constants in a cubic phase within a multiphase alloy - X-ray diffraction measurements and inverse scale transition modelling, J. Appl. Crystallogr. 38, 30-37 (2005b).

[34] S. Fréour, D. Gloaguen, M. François, and R. Guillén, Modelling and simulation of multi-phase effects on X-ray elasticity constants, Phys. Stat. Solidi B 239, 297-309 (2003). 
[35] R. W. Vook and F. Witt, Thermally induced strains in evaporated films, J. Appl. Phys. 36, 2169-2171 (1965).

[36] U. Welzel, M. Leoni, and E. J. Mittermeijer, The determination of stresses in thin films; modelling elastic grain interaction, Philos. Mag. 83(5), 603-630 (2003b).

[37] U. Welzel, S. Fréour, A. Kumar, and E. J. Mittermeijer, Diffraction stress analysis using direction dependent grain-interaction models, Mater. Sci. Forum 490-491, 7-12 (2005b).

[38] D. H. Chung and W. R. Buessem, The elastic anisotropy of crystals, J. Appl. Phys. 39, 217-245 (1967).

[39] V. Hauk and H. Kockelmann, Ermittlung der Einkristallkoeffizienten aus den mechanischen und röntgenographischen Elastizitätskonstanten des Vielkristalls, Z. Met.kd. 70(8), 500-502 (1979).

[40] M. Hayakawa, S. Imai, and M. Oka, Determination of single-crystal elastic constants from a cubic polycrystalline aggregate J. Appl. Crystallogr. 18, 513-518 (1985).

[41] T. Gnäupel-Herold, P. C. Brand, and H. J. Prask, Calculation of single-crystal elastic constants for cubic crystal symmetry from powder diffraction data, J. Appl. Crystallogr. 31, 929-935 (1998). 